\title{
HOMOLOGY OF CLOSED ORBITS OF ANOSOV FLOWS
}

\author{
J. F. PLANTE ${ }^{1}$
}

\begin{abstract}
It is shown that for a volume preserving Anosov flow on a compact manifold the closed orbits span the first homology (with real coefficients). The proof uses the notion of asymptotic cycles and results concerning the space of invariant measures for hyperbolic flows.
\end{abstract}

0 . Introduction. Let $M$ be a compact Riemannian manifold which has a smooth volume element $\eta$. Let $\varphi_{t}: M \rightarrow M$ be a $\mathscr{C}^{\infty}$ Anosov flow which leaves $\eta$ invariant. It is known [1] that for such a flow the union of periodic orbits is dense in $M$. The purpose of this note is to show that if $K \subset M$ is the union of closed orbits of $\varphi_{t}$ then the induced map on homology $H_{1}(K ; \boldsymbol{R}) \rightarrow H_{1}(M ; R)$ is surjective. (Actually, somewhat more is proved; see (2.1).) The proof given here depends heavily on a result of Sigmund [8] which, in turn, is based on results of Bowen [3]. For adequate background on the subject of Anosov flows see [1], [4], [6], [9].

1. The winding cycle. For any smooth divergence-free vector field $X$ on $M$ (i.e., such that $L_{X} \eta=0$ ) we define the winding cycle $A(X)$ of $X$ as follows:

$$
A(X)(\vartheta)=\int_{M} \vartheta(X) \eta
$$

where $\vartheta$ is a closed one-form on $M$. It is easily seen that this definition depends only on the cohomology class of $\vartheta$ in $H^{1}(M ; R)$. If we think of $H_{1}(M ; \boldsymbol{R})$ as being $\operatorname{Hom}\left(H^{1}(M ; \boldsymbol{R}) ; \boldsymbol{R}\right)$ then we have $A(X) \in H_{1}(M ; \boldsymbol{R})$. This definition may be extended as follows. Given any normalized measure $\mu$ on $M$ which is invariant under the $X$-flow we define the winding cycle (or asymptotic cycle [7]) $A_{\mu}$ by

$$
A_{\mu}(X)(\vartheta)=\int_{\lambda} \vartheta(X) d \mu
$$

Received by the editors February 15, 1972.

AMS (MOS) subject classifications (1970). Primary 58F15.

Key words and phrases Anosov flow, homology, closed orbit, invariant measure, asymptotic cycle.

${ }^{1}$ Research supported by National Science Foundation grant GP-28267A1.

(c) American Mathematical Society 1973 
Denote the $X$-flow by $\alpha_{t}$. Since we have

$$
\begin{aligned}
A_{\mu}(X)(d f) & =\int_{M} \lim _{t \rightarrow 0} \frac{f\left(\alpha_{t}(p)\right)-f(p)}{t} d \mu(p) \\
& =\lim _{t \rightarrow 0} \frac{1}{t}\left[\int_{M} f\left(\alpha_{t}(p)\right) d \mu(p)-\int_{M} f(p) d \mu(p)\right]=0
\end{aligned}
$$

(by the Lebesgue bounded convergence theorem and $\alpha_{t}$-invariance of $\mu$ ), we conclude that $A_{\mu}$ represents an element of $H_{1}(M ; R)$. Further discussion of these concepts may be found in [2], [5], [7].

Let $\mathfrak{X}$ denote the space of $\mathscr{C}^{\infty}$-vector fields on $M$ and

$$
\mathfrak{X}_{\eta}=\left\{X \in \mathfrak{X} \mid L_{X} \eta=0\right\} .
$$

1.1 LemMA (ARNOLd [2]). $A: \mathfrak{X}_{\eta} \rightarrow H_{1}(M ; R)$ is a surjective linear map.

Proof. We have $A(X)(\vartheta)=\int_{M} \vartheta(X) \eta=\int_{M} \vartheta \wedge i_{X} \eta$ where $i_{X}$ denotes interior multiplication by $X$. However, the map $X \rightarrow i_{X} \eta$ is a one-one correspondence between $\mathfrak{X}_{\eta}$ and the space of closed $(n-1)$-forms where $n=$ dimension $M$. (Note that $d i_{X} \eta=L_{X} \eta=0$.) Since $\int_{M} \vartheta \wedge i_{X} \eta$ is the usual pairing of the cohomology classes of $\vartheta$ and of $i_{X} \eta$ the result follows.

Now let $C(M)$ denote the Banach space of continuous real valued functions on $M$. In the usual way, $\varphi_{t}$-invariant measures on $M$ are thought of as elements of $C^{*}(M)$ (the dual space of $C(M)$ ). The following is a special case of a result of Sigmund [8] (see also [3]).

1.2 THEOREM. If $\varphi_{t}: M \rightarrow M$ is a volume preserving Anosov flow then the set of closed orbit measures (i.e., the invariant measures having a single closed orbit as support) is dense in the set of invariant measures in the weak* topology.

2. The main result. Consider now the linear map $\bar{A}: C_{I}^{*}(M) \rightarrow H_{1}(M ; R)$ defined by $\bar{A}(\mu)=A_{\mu}$ where we are considering a fixed $\mathscr{C}^{\infty}$ flow on $M$ and $C_{I}^{*}(M)$ denotes the subspace of $C^{*}(M)$ spanned by the invariant measures. $\bar{A}$ is continuous in the weak* topology on $C^{*}(M)$. Let $I \subset C^{*}(M)$ denote the set of normalized invariant measures. $\bar{A}(I)$ is a compact convex subset of the finite dimensional space $H_{1}(M ; R)$.

2.1 TheOREM. Let $\varphi_{t}: M \rightarrow M$ be a smooth volume preserving Anosov flow. Then: $\bar{A}(I)$.

(i) The set of $A_{\mu}$ where $\mu$ is a closed orbit measure is (weak*) dense in 
(ii) The cone through $\bar{A}(I)$ in $H_{1}(M ; R)$ contains an open subset of $H_{1}(M ; R)$.

(iii) If $K \subset M$ is the set of closed orbits of $\varphi_{t}$ then the induced map $H_{1}(K ; \boldsymbol{R}) \rightarrow H_{1}(M ; R)$ is surjective.

Proof. (i) follows from (1.2) and continuity of $\bar{A}$. To prove (ii) let $X=(d / d t) \varphi_{t}$ and $\mathscr{N}$ be a neighborhood of $X$ in $\mathfrak{X}_{\eta}$ (with $\mathscr{C}^{1}$-topology) such that:

(a) If $Z \in \mathscr{N}$ then the $Z$-flow is Anosov and is conjugate to $\varphi_{t}$ (not necessarily preserving parametrization) by a homeomorphism $h_{z}: M \rightarrow M$ which is a differentiable on orbits [4].

(b) $A(\mathscr{N})$ contains an open neighborhood of $A(X)$ in $H_{1}(M ; R)$.

(a) is structural stability of Anosov flows and (b) is just (1.1). Thus, $h_{z}$ is a conjugacy (preserving parametrization) between the $Z$-flow and a differentiable reparametrization of $\varphi_{t}$. Specifically, there is a continuous function $f_{z}: M \rightarrow R^{+}$such that $\left(h_{z}\right)_{*}(Z)=f_{z} X$. Let $\mu_{z}$ be the measure defined by $\mu_{z}(S)=\int_{h_{z}-1(S)} \eta$ where $S \subset M$ is a Borel set and we think of $\eta$ as being the measure determined by the volume form. Now the measure $f_{z} \mu_{z}$ is $\varphi_{t}$-invariant and $\bar{A}\left(f_{z} \mu_{z}\right)=A(Z)$. Since $A(\mathscr{N}) \subset H_{1}(M ; R)$ contains an open set, (ii) follows. (iii) follows from (ii) and (1.2). This completes the proof of (2.1).

3. Remarks. If $\varphi_{t}$ is the geodesic flow on the unit tangent bundle of a compact manifold of negative curvature then it is known that $A(X)=0$ [1], [6]. In such a case we see that $\bar{A}(I)$ itself is the closure of a convex open set in $H_{1}(M ; R)$. On the other hand, if $\varphi_{t}$ is the suspension of an Anosov diffeomorphism then $\bar{A}(I)$ has codimension one in $H_{1}(M ; R)$ and, in particular, does not contain an open set. It seems likely that this is the only case in which $\bar{A}(I)$ does not contain an open set. The results of [3] should be useful in this regard. The main result (2.1) should also be true if we assume that, instead of being volume preserving, $\varphi_{t}$ has $M$ as its nonwandering set. A different proof (one that does not involve (1.1)) will be necessary.

\section{REFERENCES}

1. D. V. Anosov, Geodesic flows on closed Riemann manifolds with negative curvature, Trudy Mat. Inst. Steklov. 90 (1967)=Proc. Steklov Inst. Math. 90 (1967). MR 39 \#3527.

2. V. I. Arnol'd, The one-dimensional cohomologies of the Lie algebra of divergencefree vector fields, and the winding numbers of dynamical systems, Funkcional Anal. $\mathrm{i}$ Priložen. 3 (1969), no 4, 77-78. (Russian) MR 41 \#7706.

3. R. Bowen, Periodic orbits for hyperbolic flows, Amer. J. Math. 94 (1972), 1-30.

4. M. W. Hirsch, C. C. Pugh and M. Shub, Invariant manifolds, Bull. Amer. Math. Soc. 76 (1970), 1015-1019. 
5. J. Plante, Diffeomorphisms with invariant line bundles, Invent. Math. 13 (1971), 325-334.

6. - Anosov flows, Amer. J. Math. 94 (1972).

7. S. Schwartzman, Asymptotic cycles, Ann. of Math. (2) 66 (1957), 270-284. MR $19,568$.

8. K. Sigmund, On the space of invariant measures for hyperbolic flows, Amer. J. Math. 94 (1972), 31-37.

9. S. Smale, Differentiable dynamical systems, Bull. Amer. Math. Soc. 73 (1967), 747-817. MR 37 \#3598.

Department of Mathematics, University of Wisconsin, Madison, Wisconsin 53706 